\title{
Determinants of the Quality of the Cesarean Section in Mbuji-Mayi City (Democratic Republic of Congo)
}

\author{
Balela Kabasu Marie Claire1 ${ }^{*}$, Mbuyi Mukendi Fortunat1, Kabunda Mbala John1, \\ Kadima Cibangu' ${ }^{2}$, Kakese Tshimanga ${ }^{3}$, Kabamba Nzaji Michel ${ }^{4}$, \\ Kapanga Kapita Sébastien5, Beya Beya Laurent-Blaise5, Jean Cicéron Mbunda Mulowayi'
}

${ }^{1}$ Public Health Option, Community Health Section, Higher Institute of Medical Techniques of Mbuji-Mayi, Mbuji-Mayi,
Democratic Republic of Congo
${ }^{2}$ Nursing Section, Higher Institute of Medical Techniques of Mbuji-Mayi, Mbuji-Mayi, Democratic Republic of Congo
${ }^{3}$ Medicine Faculty, University of Mwene-Ditu, Lomami, Democratic Republic of Congo
${ }^{4}$ Medicine Faculty, University of Kamina, Kamina, Democratic Republic of Congo
${ }^{5}$ Laboratory Technical Section, Higher Institute of Medical Techniques of Mbuji-Mayi, Mbuji-Mayi, Democratic Republic of Congo
${ }^{6}$ Office of Health Information Communication and Research, Provincial Health Division, Mbuji-Mayi, Democratic Republic of
Congo
Email: *ebenkabunda@gmail.com

How to cite this paper: Claire, B.K.M., Fortunat, M.M., John, K.M., Cibangu, K., Tshimanga, K., Michel, K.N., Sébastien, K.K., Laurent-Blaise, B.B. and Mbunda Mulowayi, J.C. (2021) Determinants of the Quality of the Cesarean Section in Mbuji-Mayi City (Democratic Republic of Congo). Open Access Library Journal, 8: e6307. https://doi.org/10.4236/oalib.1106307

Received: April 7, 2020

Accepted: February 23, 2021

Published: February 26, 2021

Copyright () 2021 by author(s) and Open Access Library Inc.

This work is licensed under the Creative Commons Attribution International License (CC BY 4.0).

http://creativecommons.org/licenses/by/4.0/

(c) (i) Open Access

\begin{abstract}
Objective: The overall objective of this study was to make a small contribution to improving the health of mothers and children. Method: This is a transversal descriptive study, which took place in the city of Mbuji-Mayi, exactly in the general reference hospitals of this city during the period from March 13 to June 12, 2017, on a sample of 103 cases of cesarean sections. The determinants of the quality of the cesarean section were found by the bivariate analyzes on SPSS. Results: After collecting and analyzing data, we arrived at the results according to which: The cesarean section rate is $8.9 \%$, the fairly good, low and medium quality cesarean section represented 51.5\%, $2.9 \%$ and $45.6 \%$ respectively and if the operating room was available, the probability of having a mid-quality cesarean was 90 times higher $(\mathrm{p}<0.001)$, 14 times higher in the case of a mother and child alive than in the event of death of the child $(\mathrm{p}<0.001)$ and 4.3 times weak or fairly good with an ineffective kit $(\mathrm{p}<0.001)$. Conclusion: The cesarean section rate is $8.9 \%$ and the determinants of quality cesarean section were: the availability of the operating room, mother-child prognosis and the availability of the operating kit. These results will help decision-makers in health matters to train and retrain health personnel, particularly midwives, on the benefits of prenatal consultation and its objectives, timely referral, and on the quality of work supervision.
\end{abstract}




\section{Subject Areas}

Gynecology \& Obstetrics

\section{Keywords}

Determinants, Quality, The Cesarean Section

\section{Introduction}

Maternal and child health $(\mathrm{MCH})$ is both an indicator and an essential factor for socio-economic and human development. It is also a fundamental human right. This concept occupies a central place in the Millennium Development Goals (MDGs) and is taken up in the Sustainable Development Goals (SDGs) [1]. However, the health situation of the mother and child is still worrying. Indeed, since the $20^{\text {th }}$ century, the United Nations estimates that more than $10,000,000$ women die from complications related to pregnancy and childbirth. The World Health Organization [2] maintains that 1500 women die every day from complications linked to pregnancy and childbirth such as: abortions, premature deliveries, postpartum hemorrhages, vesico-vaginal fistulas, maternal death etc. In 2005, she reported that Asia and Africa together accounted for approximately $95 \%$ of maternal deaths and almost $90 \%$ of neonatal deaths, representing a mortality rate of 300 to 1500 cases per 100,000 births live versus less than 10 cases in developed countries.

To manage these maternal complications, clinicians administer emergency obstetric care, including cesareans. These clinicians attribute benefits such as preventing vaginal or perineal damage, avoiding an emergency cesarean, and the risks of unsuccessful vaginal birth. Despite the benefits they offer to patients, newborns and families, these surgical techniques also have pathological repercussions on the health of the mother and child, as well as social problems [3].

The WHO group of experts, meeting in Geneva on October $8^{\text {th }}$ and $9^{\text {th }}$ days, 2014, made the recommendations related to the practice of cesarean section: the quality of care, particularly in terms of safety, remains an important factor to consider when analyzing cesarean and mortality rates; the risk of infection and complications due to surgery are potentially dangerous, particularly in places that do not have the infrastructure and/or capacity necessary to guarantee surgical safety.

The practice of cesarean section has been the subject of controversy and discussion for several years. Its frequency has increased very rapidly in the medicalized countries of Europe and North America [4] [5], but also in certain developing or emerging countries, for example Brazil where the figures reach or even exceed $50 \%$ of the frequency of cesarean section.

These high frequencies have given rise to numerous evaluative studies in 
the world, defining the determinants of cesarean section, in particular on: the risks and benefits, the causes of the inflation of this intervention, the legitimacy of some of its indications, the differences between the Cesarean sections in emergency and those which are planned, the Cesarean section techniques without forgetting the outcome of the Cesarean sections in terms of maternal and perinatal morbidity and mortality linked to the quality of these.

Often, despite the urgency of the cesarean, there are almost rapid execution problems, the consequences of which are uterine rupture, a serious situation endangering the life of the fetus and the mother.

The multifaceted crises that the DRC has experienced have affected all sectors, particularly the health sector. A study on the cesarean section and especially on the determinants of its quality in the General Reference Hospitals of the city of Mbuji-Mayi would not be without interest.

The overall objective of this study was to make a small contribution to improving the health of mothers and children. She set herself the following specific objectives:

- Determine the frequency of the cesarean;

- Identify the characteristics of women under cesarean;

- To determine the maternal and fetal prognosis of the cesarean operation.

\section{Material and Method}

\subsection{A Framework}

Our study was conducted in the Democratic Republic of Congo, specifically in the diamond town of Mbuji-Mayi which is the head town of the province of Kasaï Oriental.

\subsection{Type of Study}

This is a cross-sectional descriptive study, which took place in the city of Mbuji-Mayi, precisely in all general hospitals of reference of this city during the period from 13 March to 12 June 2017 is for 3 months.

\subsection{Study Population}

The population of our study consists of all parturients admitted to Gyneco-obstetrics services in all general hospitals of reference in the city of Mbuji-Mayi for the diagnosis of cesarean section. Scheduled cesareans were also considered.

\section{- Selection criteria}

a) Inclusion criteria:

Included in this study are:

$>$ The parturients whose cesarean section was given and whose operation took place in one of the HGR maternity wards in the city of Mbuji-Mayi;

Newborns from these cesarean sections; 
All cesarean women who agreed to participate in the study;

Having given birth in one of the maternity wards of the hospitals targeted by our study.

b) Non-inclusion criteria:

$>$ Parturients operated in another structure and sent for care;

Laparotomies for frank uterine rupture with expulsion of the conception product into the large abdominal cavity.

\subsection{Data Collection and Analysis}

The study was submitted to the authority of the chief of the provincial health division of Kasaï Oriental and the managers of the general reference hospitals (GRH) (Doctors Chief of the health zones and administrators managers of the GRH). All information relating to women under cesarean section was confidential and accessible only to the investigator and the research team.

The data was subjected to quality control which consisted of regular verification and validation every day. They were collected and processed using Epiinfo 2007 software, SPSS and Excel 2007 which allowed us to carry out descriptive analyzes and those of various kinds.

The following statistical measurements were carried out: the frequencies for the qualitative variables and the means, the standard deviation, the Odd ratio (OR) and its confidence intervals (IC) for the quantitative variables,

To assess the quality of the Cesarean section, we proceeded by 2 approaches:

\section{$1^{\text {st }}$ Approach:}

Given our local conditions, we have selected a number of process and result indicators that meet the requirements and expectations for performing a quality cesarean.

The conditions of treatment: the period of treatment, the availability of the kit, the availability of the nursing staff, the availability of the operating room and the availability of blood.

Carrying out and results of the intervention: the quality of the operator, the quality of the anesthesia, the relevance of the operating indications, intraoperative complications, the life of the mother and child and the APGAR from newborn to birth.

Post-operative follow-up: compliance with the post-operative protocol, immediate post-operative surveillance, post-operative complications, length means of hospital stay and score scoring ( 0 to 3 ) was done for the criteria identified above.

\section{$2^{\text {nd }}$ Approach:}

The quality level of the cesarean sections was determined from the scores obtained during the performance of the discriminating variables.

The Analysis focused on a scale where caesareans are said:

"Good Quality" if all the discriminatory variables have been performed;

"Average" and "Poor or fairly good" if at least one of the discriminatory variables is not achieved. 


\subsection{Ethical Considerations}

In contacts with women under cesarean section, an investigator explained to them the merits of the research by giving them the assurance of anonymity in their statements as well as the confidentiality of the information recorded on the collection instruments provided by the women participants to obtain their free consent. The investigator explained to the participants the importance of the study and the results will only be used for scientific purposes.

\section{Results}

\subsection{Descriptive Analysis}

As shown in Table 1, the cesarean section rate was $8.9 \%$, the mean age of our respondents was $28.9 \pm 7.2$ years (the age group 20 to 35 years more represented with $65 \%$, the mean parity was $4.0 \pm 3.2$ children (the pauciparous were more represented with $29.1 \%$ each), the gravidity's mean was $4.7 \pm 4.3$ pregnancies (the large multigests took the lead with $28.2 \%, 15,5 \%$ of caesareans had a history of abortion and $17.5 \%$ had a scar uterus.

Table 2 shows that $98.0 \%$ of women with cesarean section had followed Prenatal consultations and $52.4 \%$ of them had performed 4 sessions or more, in $90.3 \%$ of cases hemoglobin was performed, $64.1 \%$ of women respondents had been vaccinated, $89.3 \%$ had not suffered during pregnancy, $93.2 \%$ had not had a hypertension attack and $96.1 \%$ had not had anemia during pregnancy.

It should be noted in Table 3 that $95.1 \%$ of women surveyed had a good general condition, the mean of uterine height was $33.4 \pm 3.7 \mathrm{~cm}(71.8 \%$ had less than $36 \mathrm{~cm}$ ) and the mean gestational age was $38.5 \pm 2.4$ weeks of amenorrhea (in $77.7 \%$ of cases was between 37 - 42 weeks), $53.4 \%$ of the caesareans had regular contractions and the fetal movements were active at $84.5 \%$, the noises of the fetal hearts were regular in $73.8 \%$ of cases, cesarean section was performed in an emergency at $89.3 \%$, the indication that came to mind was the transverse presentation with $32.0 \%$ and $90.3 \%$ of women had a pelvis normal.

Table 4 reveals that the fetal presentation was cephalic at $68.9 \%$, of which $62.1 \%$

Table 1. Cesarean section rate and characteristics of the parturient.

\begin{tabular}{ccc}
\hline Variables & Workforce $(\mathrm{n}=103)$ & $\%$ \\
\hline Cesarean section frequency & 103 & 8.9 \\
Cesarean & 1150 & 91.1 \\
Eutocic delivery & & \\
Woman's age (years) & 12 & 11.7 \\
$<20$ & 67 & 65.0 \\
20 to 35 & 24 & 23.3 \\
$>35$ & $\mathbf{2 8 . 9}$ & \\
Means & &
\end{tabular}




\section{Continued}

\begin{tabular}{|c|c|c|}
\hline Parity & & \\
\hline Primiparous & 27 & 26.2 \\
\hline Pauciparous & 30 & 29.1 \\
\hline Multiparous & 23 & 22.3 \\
\hline Large multiparous & 23 & 22.3 \\
\hline Mean & $4.0 \pm 3.2$ & \\
\hline \multicolumn{3}{|l|}{ Gravidity } \\
\hline Gravida & 26 & 25.2 \\
\hline Paucigestes (2 - 3) & 26 & 25.2 \\
\hline Multigravidae (4 - 5) & 22 & 21.4 \\
\hline Multigravidae large $(\geq 6)$ & 29 & 28.2 \\
\hline Mean & $4.7 \pm 4.3$ & \\
\hline \multicolumn{3}{|l|}{ Abortion } \\
\hline Yes & 16 & 15.5 \\
\hline No & 87 & 84.5 \\
\hline \multicolumn{3}{|l|}{ History of Cesarean } \\
\hline Yes & 18 & 17.5 \\
\hline No & 85 & 82.5 \\
\hline
\end{tabular}

Table 2. Features related to pregnancy monitoring.

\begin{tabular}{ccc}
\hline Variables & Workforce $(\mathrm{n}=103)$ & $\%$ \\
\hline Follow EIC & 101 & 98.0 \\
Yes & 2 & 2.0 \\
No & & \\
Number of CPN & 47 & 47.6 \\
$<4$ & 54 & 52.4 \\
$\geq 4$ & & \\
Hemoglobin & 10 & 9.7 \\
Yes & 93 & 90.3 \\
No & & \\
Hypertension & 7 & 6.8 \\
Yes & 96 & 93.2 \\
No & 99 & 3.9 \\
Yes & 4 & 96.1 \\
No & & \\
\hline
\end{tabular}


Table 3. Characteristics linked to the admission diagnosis.

\begin{tabular}{|c|c|c|}
\hline Variables & Workforce $(n=103)$ & $\%$ \\
\hline \multicolumn{3}{|l|}{ General condition } \\
\hline Well & 98 & 95.1 \\
\hline Weathered & 5 & 4.9 \\
\hline \multicolumn{3}{|l|}{ Uterine height } \\
\hline$<36 \mathrm{~cm}$ & 74 & 71.8 \\
\hline$\geq 36 \mathrm{~cm}$ & 29 & 28.2 \\
\hline Mean & $33.4 \pm 3.7$ & \\
\hline \multicolumn{3}{|l|}{ Gestational age in Amenorrhea Weeks } \\
\hline$<37$ & 20 & 19.4 \\
\hline 37 to 42 & 80 & 77.7 \\
\hline$>42$ & 3 & 2.9 \\
\hline Mean & $38.5 \pm 2.4$ & \\
\hline \multicolumn{3}{|l|}{ Uterine contractions } \\
\hline Regular & 55 & 53.4 \\
\hline Irregular & 36 & 35.0 \\
\hline Absent & 12 & 11.7 \\
\hline \multicolumn{3}{|l|}{ Cesarean section indications } \\
\hline Stationary expansion & 1 & 1.0 \\
\hline Placenta prævia & 9 & 8.7 \\
\hline Headquarters presentation & 7 & 6.8 \\
\hline Cross-functional presentation & 33 & 32.0 \\
\hline Cord procidence & 1 & 1.0 \\
\hline Dynamic dystocia & 9 & 8.7 \\
\hline Fetal suffering & 15 & 14.6 \\
\hline Pelvic fetal disproportion & 14 & 13.6 \\
\hline Eclampsia & 1 & 1.0 \\
\hline Other & 13 & 12.6 \\
\hline \multicolumn{3}{|l|}{ Basin condition } \\
\hline Normal & 93 & 90.3 \\
\hline Unnatural & 10 & 9.7 \\
\hline \multicolumn{3}{|l|}{ Fetal movements } \\
\hline Active & 87 & 84.5 \\
\hline Not active & 16 & 15.5 \\
\hline \multicolumn{3}{|l|}{$\mathrm{BCF}$} \\
\hline Regular & 78 & 73.8 \\
\hline Irregular & 8 & 7.8 \\
\hline Imperceptible or absent & 19 & 18.4 \\
\hline \multicolumn{3}{|l|}{ Type of Cesarean } \\
\hline Emergency & 92 & 89.3 \\
\hline Scheduled & 10 & 9.7 \\
\hline
\end{tabular}


Table 4. Distribution of data according to characteristics.

\begin{tabular}{|c|c|c|}
\hline Variables & Workforce $(n=103)$ & $\%$ \\
\hline \multicolumn{3}{|c|}{ Fetal presentation } \\
\hline Cephalic & 71 & 68.9 \\
\hline Seat & 7 & 6.8 \\
\hline Transverse & 25 & 24.3 \\
\hline \multicolumn{3}{|c|}{ Degree of commitment } \\
\hline Yes & 64 & 62.1 \\
\hline No & 39 & 37.9 \\
\hline \multicolumn{3}{|c|}{ Erasing the cervix } \\
\hline Yes & 98 & 95.1 \\
\hline No & 5 & 4.9 \\
\hline \multicolumn{3}{|c|}{ Expansion phase } \\
\hline Active & 61 & 59.2 \\
\hline Latency & 42 & 40.8 \\
\hline \multicolumn{3}{|c|}{ State of membranes } \\
\hline Broken & 42 & 40.8 \\
\hline Intact & 61 & 59.2 \\
\hline \multicolumn{3}{|c|}{ Appearance of Amniotic Liquid } \\
\hline Clear & 60 & 58.3 \\
\hline Meconial & 13 & 12.6 \\
\hline Tinted & 16 & 15.5 \\
\hline Bloody & 14 & 13.6 \\
\hline
\end{tabular}

were engaged, the cervix was obliterated at $97.1 \%$ in most cases, $59.2 \%$ of the respondents were during the active phase of their dilation, at $59.2 \%$ the membranes were intact and the appearance of the Amniotic Liquid was clear at 58.3\%

Table 5 indicates that $48 \%$ of cesarean women were evacuated in emergency, $60.2 \%$, had come by motorbike, the mean cost of cesarean section was $214260.7 \pm$ $99157.4 \mathrm{FC}$ ( $233 \pm 107$ USD), the mean time between reference and admission was $71.3 \pm 46.3$ minutes, the mean time between admission and decision was $87.3 \pm$ 214.6 minutes, the time between decision and intervention was on mean $55.3 \pm 80.9$ minutes and the mean intervention time was $63.5 \pm 21.2$ minutes.

These results (Table 6) show that the cesarean section was performed by the general practitioner in $98.1 \%$ of the cases and under general anesthesia in $98.1 \%$, $98.1 \%$ of them were performed without incident, $73.8 \%$ of respondents didn't benefit from induction antibiotic therapy, $97.1 \%$ of the blood was not available and the operating kit was available in $37.9 \%$ of cases, the postoperative continuation was $91.3 \%$ simple, at $100 \%$ the maternal prognosis was good, and the means 
Table 5. Distribution of data according to accessibility to reference maternity.

\begin{tabular}{|c|c|c|}
\hline Variables & Workforce $(n=103)$ & $\%$ \\
\hline \multicolumn{3}{|c|}{ Mode of admission } \\
\hline One came & 42 & 40.8 \\
\hline Reference & 11 & 10.7 \\
\hline Evacuated & 50 & 48.5 \\
\hline \multicolumn{3}{|c|}{ The mode of transport } \\
\hline Motorbike & 62 & 60.2 \\
\hline \multicolumn{3}{|l|}{ Cost of Cesarean } \\
\hline$<100,000 \mathrm{FC}$ & 4 & 3.9 \\
\hline$\geq 100,000 \mathrm{FC}$ & 99 & 96.1 \\
\hline Mean & $214,260.7 \pm 99,157.4 \mathrm{FC}$ & \\
\hline \multicolumn{3}{|c|}{ Time between reference and admission } \\
\hline$<60$ minutes & 23 & 22.3 \\
\hline$\geq 60$ minutes & 80 & 77.7 \\
\hline Mean & $71.3 \pm 46.3$ minutes & \\
\hline \multicolumn{3}{|c|}{ Delay between admission and decision } \\
\hline$<60$ minutes & 89 & 86.4 \\
\hline$\geq 60$ minutes & 14 & 13.6 \\
\hline Mean & $87.3 \pm 214.6$ minutes & \\
\hline \multicolumn{3}{|c|}{ Time between decision and intervention } \\
\hline$<60$ minutes & 89 & 86.4 \\
\hline$\geq 60$ minutes & 14 & 13.6 \\
\hline Mean & $55.3 \pm 80.9$ minutes & \\
\hline \multicolumn{3}{|c|}{ Duration of intervention } \\
\hline$<60$ minutes & 50 & 48.5 \\
\hline$\geq 60$ minutes & 53 & 51.5 \\
\hline Mean & $63.5 \pm 21.2$ minutes & \\
\hline
\end{tabular}

Table 6. Characteristics linked to the surgical act and post-operative monitoring.

\begin{tabular}{ccc}
\hline Variables & Workforce $(\mathbf{n}=103)$ & $\%$ \\
\hline Block availability & 49 & 47.6 \\
Available & 54 & 52.4 \\
Not available & & \\
Qualification of the surgeon & 2 & 1.9 \\
Obstetrician & 101 & 98.1 \\
General practitioner & & \\
Type of anesthesia & &
\end{tabular}




\section{Continued}

\begin{tabular}{|c|c|c|}
\hline General & 101 & 98.1 \\
\hline Locoregional & 2 & 1.9 \\
\hline \multicolumn{3}{|l|}{ Incident during the intervention } \\
\hline Yes & 2 & 1.9 \\
\hline No & 102 & 98.1 \\
\hline \multicolumn{3}{|l|}{ Induction antibiotic therapy } \\
\hline Yes & 27 & 26.2 \\
\hline No & 76 & 73.8 \\
\hline \multicolumn{3}{|l|}{ Blood availability } \\
\hline Blood product available as needed & 3 & 2.9 \\
\hline Blood product not available as needed & 100 & 97.1 \\
\hline \multicolumn{3}{|l|}{ Availability of the operating kit } \\
\hline Available & 39 & 37.9 \\
\hline Not available & 64 & 62.1 \\
\hline \multicolumn{3}{|l|}{ Post-operative follow-up } \\
\hline Simple & 94 & 91.3 \\
\hline Complicated & 9 & 8.7 \\
\hline \multicolumn{3}{|l|}{ Maternal prognosis } \\
\hline Well & 103 & 100.0 \\
\hline \multicolumn{3}{|l|}{ Duration of hospitalization } \\
\hline$\geq 7$ days & 103 & 100.0 \\
\hline Means & $18.3 \pm 6.4$ days & \\
\hline
\end{tabular}

hospital stay was $18.3 \pm 6.4$ days.

Table 7 indicates that $97.1 \%$ of the newborns were in good condition at birth, among them $63.1 \%$ were not resuscitated, their means weight was $3244.8 \pm 729.3$ grams (those who had normal weight led with 78.6\%) most newborns had no birth defect in $99.0 \%$ of cases and the prognosis for the newborn was $79.6 \%$ satisfactory.

Table 8 shows that $51.5 \%$ of the cesarean sections had a fairly good quality while $45.6 \%$ had an average quality.

\subsection{Bivariate Analysis Results}

Analysis of this Table 9 shows that there is not a relationship between socio-demographic characteristics and quality cesarean section. The method of admission does not influence the quality cesarean section ( $p>0.05)$.

It should be noted in Table 10 that gestational age, dilation, condition of the water pocket as well as the type of cesarean are not significantly related to quality 
Table 7. Characteristics linked to the newborn.

\begin{tabular}{|c|c|c|}
\hline Variables & Effectif $(n=103)$ & $\%$ \\
\hline \multicolumn{3}{|c|}{ State of the newborn at birth } \\
\hline Well & 3 & 2.9 \\
\hline Bad & 100 & 97.1 \\
\hline \multicolumn{3}{|c|}{ Resuscitation of the newborn } \\
\hline Yes & 38 & 36.9 \\
\hline No & 65 & 63.1 \\
\hline \multicolumn{3}{|c|}{ Weight of newborn (grams) } \\
\hline Normal weight & 81 & 78.6 \\
\hline Low weight & 10 & 9.7 \\
\hline Macrosomia & 12 & 11.7 \\
\hline Means & $3244.8 \pm 729.3$ grams & \\
\hline \multicolumn{3}{|l|}{ APGAR 5} \\
\hline$<7$ & 61 & 59.2 \\
\hline$\geq 7$ & 42 & 40.8 \\
\hline \multicolumn{3}{|c|}{ Congenital malformation } \\
\hline Yes & 1 & 1.0 \\
\hline No & 102 & 99.0 \\
\hline \multicolumn{3}{|c|}{ Newborn prognosis } \\
\hline Satisfactory & 82 & 79.6 \\
\hline Bad & 21 & 204 \\
\hline
\end{tabular}

Table 8. Distribution of cases by quality of cesarean section.

\begin{tabular}{ccc}
\hline Quality of cesarean section & Workforce & $\%$ \\
\hline Pretty good & 53 & 51.5 \\
Low & 3 & 2.9 \\
Average & 47 & 45.6 \\
Total & 103 & 100.0 \\
\hline
\end{tabular}

Table 9. Socio-demographic characteristics of women and those related to maternity access.

\begin{tabular}{|c|c|c|c|c|}
\hline \multirow[b]{2}{*}{ Variables } & \multicolumn{2}{|c|}{ Bundled Cesarean Quality } & \multirow[b]{2}{*}{ OR/IC } & \multirow[b]{2}{*}{$\mathrm{p}$} \\
\hline & $\begin{array}{l}\text { Poor or fairly } \\
\operatorname{good}(n=56)\end{array}$ & $\begin{array}{l}\text { Average } \\
(n=47)\end{array}$ & & \\
\hline \multicolumn{5}{|l|}{ Woman's age } \\
\hline$<20$ years & $8(66.7 \%)$ & $4(33.3 \%)$ & $0.44(0.12-1.5)$ & 0.206 \\
\hline $20-35$ years & $34(50.7 \%)$ & $33(49.3 \%)$ & & \\
\hline$>35$ years & $14(58.3 \%)$ & $10(41.7 \%)$ & $1.23(0.48-3.10)$ & 0.656 \\
\hline
\end{tabular}




\section{Continued}

\begin{tabular}{|c|c|c|c|c|}
\hline Parity & & & & \\
\hline Nulliparous & $2(66.7 \%)$ & $1(33.3 \%)$ & $0.46(0.16-1.33)$ & 0.152 \\
\hline Primiparous & $17(63.0 \%)$ & $10(37.0 \%)$ & & \\
\hline Pauciparous & $12(44.4 \%)$ & $15(55.6 \%)$ & & \\
\hline Multiparous & $11(47.8 \%)$ & $12(52.2 \%)$ & & \\
\hline Large multiparous & $14(60.9 \%)$ & $9(39.1 \%)$ & $1.48(0.57-3.86)$ & 0.413 \\
\hline \multicolumn{5}{|l|}{ Gravidity } \\
\hline Gravida & $16(61.5 \%)$ & $10(38.5 \%)$ & $0.76(0.37-1.65)$ & 0.494 \\
\hline Paucigestes & $14(53.8 \%)$ & $12(46.2 \%)$ & & \\
\hline Multigravidae & $9(40.9 \%)$ & $13(59.1 \%)$ & & \\
\hline Multigravidae large & $17(58.6 \%)$ & $12(41.4 \%)$ & & \\
\hline \multicolumn{5}{|l|}{ History of Cesarean } \\
\hline Yes & $8(44.4 \%)$ & $10(55.6 \%)$ & $0.617(0.22-1.72)$ & 0.352 \\
\hline No & $48(56.5 \%)$ & $37(43.5 \%)$ & & \\
\hline \multicolumn{5}{|l|}{ Condition } \\
\hline Well & $53(54.1 \%)$ & $45(45.9 \%)$ & $1.27(0.20-7.26)$ & 0.795 \\
\hline weathered & $3(60 \%)$ & $2(40 \%)$ & & \\
\hline \multicolumn{5}{|l|}{ Mode of admission } \\
\hline One came & $27(64.3 \%)$ & $15(37.7 \%)$ & $0.50(0.22-1.12)$ & 0.093 \\
\hline Evacuated & $6(54.5 \%)$ & $5(45.5 \%)$ & & \\
\hline Reference & $23(46.0 \%)$ & $27(54.0 \%)$ & $0.54(0.25-1.16)$ & 0.111 \\
\hline \multicolumn{5}{|l|}{ Mode of transport } \\
\hline Feet & $32(51.6 \%)$ & $30(48.4 \%)$ & $1.32(0.59-2.93)$ & 0.489 \\
\hline Motorbike & $23(59.0 \%)$ & $16(41.0 \%)$ & & \\
\hline Bike & $1(50.0 \%)$ & $1(50.0 \%)$ & & \\
\hline \multicolumn{5}{|c|}{$\begin{array}{l}\text { Delay between admission and } \\
\text { decision }\end{array}$} \\
\hline$<60$ minutes & $48(53.9 \%)$ & $41(46.1 \%)$ & $0.87(0.28-2.73)$ & 0.823 \\
\hline$\geq 60$ minutes & $8(57.1 \%)$ & $6(42.9 \%)$ & & \\
\hline \multicolumn{5}{|c|}{$\begin{array}{c}\text { Time between decision and } \\
\text { intervention }\end{array}$} \\
\hline$<60$ minutes & $52(58.4 \%)$ & $37(41.6 \%)$ & $0,87(0.28-2.74)$ & 0.037 \\
\hline$\geq 60$ minutes & $4(28.6 \%)$ & $10(71.4 \%)$ & & \\
\hline \multicolumn{5}{|c|}{ Duration of intervention } \\
\hline$<60$ minutes & $26(52.0 \%)$ & $24(48.0 \%)$ & $0.831(0.38-1.81)$ & 0.639 \\
\hline$\geq 60$ minutes & $30(56.6 \%)$ & $23(43.4 \%)$ & & \\
\hline
\end{tabular}


Table 10. Characteristics related to childbirth.

\begin{tabular}{|c|c|c|c|c|}
\hline \multirow[b]{2}{*}{ Variables } & \multicolumn{2}{|c|}{ Bundled Cesarean Quality } & \multirow[b]{2}{*}{ OR/IC } & \multirow[b]{2}{*}{$\mathrm{p}$} \\
\hline & $\begin{array}{l}\text { Poor or fairly } \\
\text { good }(n=56)\end{array}$ & $\begin{array}{l}\text { Average } \\
(n=47)\end{array}$ & & \\
\hline \multicolumn{5}{|c|}{ Gestational age (weeks) } \\
\hline$<37$ & $10(50 \%)$ & $10(50 \%)$ & $1.2(0.46-3.30)$ & 0.662 \\
\hline 37 to 42 & $44(55 \%)$ & $36(45 \%)$ & & \\
\hline$>42$ & $2(66.7 \%)$ & $1(33.3 \%)$ & $0.42(0.01-5.79)$ & 0.479 \\
\hline \multicolumn{5}{|l|}{ Dilation } \\
\hline Yes & $23(54.8 \%)$ & $19(45.2 \%)$ & $1.027(0.46-2.26)$ & 0.947 \\
\hline No & $33(54.1 \%)$ & $28(45.9 \%)$ & & \\
\hline \multicolumn{5}{|c|}{ State of the membranes } \\
\hline Broken & $27(47.4 \%)$ & $30(52.6 \%)$ & $0,528(0.23-1.16)$ & 0.112 \\
\hline Intact & $29(63.0 \%)$ & $17(37.0 \%)$ & & \\
\hline \multicolumn{5}{|c|}{ Type of Cesarean } \\
\hline Urgent & $48(52.2 \%)$ & $44(47.8 \%)$ & $0.468(0.11-1.92)$ & 0.283 \\
\hline Program & $7(70.0 \%)$ & $3(30.0 \%)$ & & \\
\hline
\end{tabular}

Table 11. Characteristics linked to the surgical procedure.

\begin{tabular}{|c|c|c|c|c|}
\hline \multirow[b]{2}{*}{ Variables } & \multicolumn{2}{|c|}{ Bundled Cesarean Quality } & \multirow[b]{2}{*}{ OR [IC to $95 \%]$} & \multirow[b]{2}{*}{$\mathrm{p}$} \\
\hline & $\begin{array}{l}\text { Poor or fairly } \\
\operatorname{good}(n=56)\end{array}$ & $\begin{array}{l}\text { Average } \\
(n=47)\end{array}$ & & \\
\hline \multicolumn{5}{|l|}{ Block availability } \\
\hline Available & $49(100 \%)$ & $0(0 \%)$ & & \\
\hline Not available & $4(7.4 \%)$ & $50(92.6 \%)$ & Indeterminate & $<0.001$ \\
\hline \multicolumn{5}{|l|}{ Blood availability } \\
\hline Available & $2(66.6 \%)$ & $1(33.3 \%)$ & $1.9(0.14-57.8)$ & 0.592 \\
\hline Not available & $51(51 \%)$ & $49(49 \%)$ & & \\
\hline \multicolumn{5}{|l|}{ Type of anesthesia } \\
\hline General & $54(53.5 \%)$ & $47(46.5 \%)$ & $0.53(0.44-0.64)$ & 0.191 \\
\hline Spinal anesthesia & $2(100.0 \%)$ & $0(0.0 \%)$ & & \\
\hline
\end{tabular}

cesarean because ( $\mathrm{p}>0.05)$.

After analyzing this Table 11, we note that the time between admission, intervention and the type of anesthesia is not significant for quality cesarean.

Table 12 shows that the availability of the block $(\mathrm{p}<0.001)$, the absence of maternal death $(\mathrm{p}<0.001)$ and the effectiveness of the operating kit $(\mathrm{p}<0.001)$ are the determinants of quality cesarean section. 
Table 12. Determinants of the quality of the cesarean section

\begin{tabular}{|c|c|c|c|c|}
\hline \multirow[b]{2}{*}{ Variables } & \multicolumn{2}{|c|}{ Bundled Cesarean Quality } & \multirow[b]{2}{*}{ OR/IC } & \multirow[b]{2}{*}{$\mathrm{p}$} \\
\hline & $\begin{array}{l}\text { Poor or fairly } \\
\text { good }(n=56)\end{array}$ & $\begin{array}{l}\text { Average } \\
(\mathrm{n}=47)\end{array}$ & & \\
\hline \multicolumn{5}{|l|}{ Care score } \\
\hline Greater than 1 hour & $3(30.0 \%)$ & $7(70.0 \%)$ & $0.32(0.08-1.33)$ & 0.103 \\
\hline Less than 1 hour & $53(57.0 \%)$ & $40(43.0 \%)$ & & \\
\hline \multicolumn{5}{|l|}{ Block availability score. } \\
\hline Available & $6(10.7 \%)$ & $43(91.5 \%)$ & $89.5(23.0-338.4)$ & $<0.001$ \\
\hline Not available & $50(89.3 \%)$ & $4(8.5 \%)$ & & \\
\hline \multicolumn{5}{|l|}{ Care staff availability score } \\
\hline Guard team present and complete & $3(33.3 \%)$ & $6(66.7 \%)$ & $0.38(0.09-1.64)$ & 0.185 \\
\hline guard team present and incomplete & $53(56.4 \%)$ & $41(43.6 \%)$ & & \\
\hline \multicolumn{5}{|l|}{ Anesthesia quality score } \\
\hline Presence of complications & $1(50.0 \%)$ & $1(50.0 \%)$ & $0.84(0.05-13.7)$ & 0.900 \\
\hline absence of complications & $55(54.5 \%)$ & $46(45.5 \%)$ & & \\
\hline \multicolumn{5}{|l|}{ Blood availability score } \\
\hline Not available & $53(53.0 \%)$ & $47(47.0 \%)$ & $0.53(0.44-0.64)$ & 0.107 \\
\hline Available & $3(100.0 \%)$ & $0(0.0 \%)$ & & \\
\hline \multicolumn{5}{|l|}{ Complication score } \\
\hline Presence & $3(33.3 \%)$ & $6(66.7 \%)$ & $0.38(0.09-1.64)$ & 0.185 \\
\hline per and postoperative absence & $53(56.4 \%)$ & $41(43.6 \%)$ & & \\
\hline \multicolumn{5}{|l|}{ Mother-child prognosis score } \\
\hline living mother and deceased child & $2(11.1 \%)$ & $16(88.9 \%)$ & $13.9(3-64)$ & $<0.001$ \\
\hline mother and child alive & $54(63.5 \%)$ & $31(36.5 \%)$ & & \\
\hline \multicolumn{5}{|l|}{ Score kit } \\
\hline Effective & $39(100 \%)$ & $0(0 \%)$ & $4,3(2.98-8.65)$ & $<0.001$ \\
\hline Ineffective & $17(30.4 \%)$ & 47 (69.6\%) & & \\
\hline \multicolumn{5}{|l|}{ Quality of Cesarean } \\
\hline Pretty good & $45(86.5 \%)$ & $7(13.5 \%)$ & $0.34(0.09-1.64)$ & 0.420 \\
\hline Low & $3(100 \%)$ & $0(0 \%)$ & & \\
\hline Average & $44(93.6 \%)$ & $3(6.4 \%)$ & & \\
\hline
\end{tabular}

\section{Discussion}

\subsection{Results of Descriptive Analyses}

In our series, the cesarean section rate was $8.9 \%$ (Table 1). This rate is within the limits set by the WHO, which recommends $5 \%$ to $15 \%$ for cesareans. However, they were lower than those found by [6] according to which in Switzerland the rate of cesarean section was above $10 \%$. On the other hand in Quebec, the rate of 
cesarean was at $24.9 \%$ in 2016 . This would be due to the fact that in the industrialized countries, the cesarean by convenience is already liberalized and many women prefer this mode of childbirth compared to the vaginal delivery.

The mean age of our respondents was $28.9 \pm 7.2$ years (Table 1 ). These results are higher than those found by [7] in his series, the mean age of the respondents was 26.1 years. This would be due to the fact that this age group is the most active for fertility.

The mean parity was $4.0 \pm 3.2$ children (Table 1 ). These results do not corroborate those found by [8] who stipulate that the mean parity was 1.6 children. And the gravidity mean was $4.7 \pm 4.3$ pregnancies (large multigravidae led 28.2\%) (Table 1). This is justified by the fact that our study is carried out in the town of Mbuji-Mayi which is an area of high popular density with a very high birth rate.

The history of abortion involved $15.5 \%$ of cesareans and $17.5 \%$ had a scar uterus (Table 1). These results are close to those of [9] who found that $18.6 \%$ of women with cesarean section had a history of cesarean section. In contrast, [8] found in their series that $40.8 \%$ of cesareans had at least one scar uterus. This would be due to the fact that in our environments, accessibility to reference care is not easy.

In terms of pregnancy monitoring, $98 \%$ of women with cesarean section had undergone $\mathrm{CPN}$ and $52.4 \%$ had had 4 or more sessions (Table 2). These results differ from those found by [8] who mention that $92.7 \%$ of cesareans had followed the CPN and $68 \%$ of them had carried out at least 3 sessions during pregnancy. This would be due to a good organization of our health system which allows all women access to the $\mathrm{CPN}$.

In $90.3 \%$ of the hemoglobin tests were carried out, $64.1 \%$ of the women surveyed had been vaccinated, $11.7 \%$ had suffered during pregnancy, $6.8 \%$ had had a hypertension attack and 3, 9\% had experienced anemia during pregnancy (Table 2). These results do not corroborate those found by [10] who in their series of less than $50 \%$ hemoglobin was performed, the caesareans were vaccinated at $54 \%, 2.4 \%$ had hypotension. This would be due to the fact that their study was carried out in the rural environment where the monitoring of the pregnant woman is weak.

General condition of women with Caesarean section on admission was good in $95.1 \%$ of cases (Table 3). These results do not agree with those of [8] who had found for their series that $82.2 \%$ of women with cesarean section were admitted in good condition.

The mean uterine height was $33.4 \pm 3.7 \mathrm{~cm}(71.8 \%$ was less than $36 \mathrm{~cm})$ (Table 3). These results are superior to those found by [9] according to which her respondents had a mean of uterine height of $31.97 \pm 3 \mathrm{~cm}$ (i $32.60 \pm 3.40 \mathrm{~cm}$ for cesareans and $31.04 \pm 2.56 \mathrm{~cm}$ for the lower tracks). These differences would be justified by the small sample size.

The mean gestational age was $38.5 \pm 2.4$ weeks of amenorrhea (in $77.7 \%$ of 
cases was between 37 - 42 weeks) (Table 3). These results are similar to those found by [10] who in their series had a gestational age mean of $38.58 \pm 2.45$ weeks. This would be a coincidence.

$53.4 \%$ of the women surveyed had regular contractions, $84.5 \%$ had active fetal movements and noises of the fetal heart were regular in $73.8 \%$ of cases (Table 3 ). These results are similar to those of [8], i.e. $87.9 \%$ of regular BCF and fetal movements were active at admission at $82 \%$.

We found that $89.3 \%$ of cesarean sections were performed in an emergency, the main indication for cesarean section was the transverse fetal presentation with $32.0 \%$ (Table 3). On the other hand [11] found acute fetal suffering (15.38\%) as the main indication. While [12] who had found in their series the indication related to cervical dystocia at $16.1 \%$, This diversity of indication would be explained by the obstetrician's concern to give birth to a child free from anoxia or trauma.

The fetal presentation was $68.9 \%$ cephalic (Table 4). On the other hand [9] found that $96.9 \%$ of children were in cephalic presentation. It would seem to be a coincidence.

According to the progression of the fetal mobile, the degree of engagement was $62.1 \%$, the cervix was obliterated at $97.1 \%$ in most cases and $59.2 \%$ of the respondents were during the active phase of their dilation (Table 4). On the other hand [10] had found that $51.96 \mathrm{~d} \%$ of women with cesarean section were during the latency phase

At admission, most women with cesarean section had 59.2\% intact membranes and the vast majority of those with ruptured ruptures, the appearance of amniotic liquid was clear at 58.3\% (Table 4). These results are lower than those found by [13] according to which the membranes were ruptured before the admission of patients into the delivery room at $65.6 \%$. On the other hand Annie F. cited by [13] had found that they were broken at $46.8 \%$. This would be due to the fact that in our environment the system of reference and counter reference suffers especially that the first level care services incarcerate the patients to earn at all costs money hence the late references.

For the most part, respondents were evacuated to emergency at $48.8 \%$ (Table 5). These results are lower than those of [8] indicating that women with cesarean section were evacuated in emergency $(61.4 \%)$. This is because in our environment evacuation is done after the failure of all the maneuvers of the first level health service with regard to emergencies and especially obstetric and neonatal ones.

The vast majority of cesarized women were evacuated by public transport (motorcycle taxi) at $60.2 \%$ (Table 5). These same results were found by [7]. It would be due to the impassability of roads in our environment which makes HGR inaccessible by vehicle transport.

The mean cost of cesarean section was 214,260.7 $\pm 99,157.4$ CF in our community ( $96.1 \%$ of cesarean sections cost more than or equal to $100,000 \mathrm{CF}$ ) 
(Table 5). These results differ from those found by [14] according to which the cost of the cesarean section in Senegal was 90,000 FCFA. This would be justified by the fact that our studies have been carried out in different environments where each health system has its regulation of negotiated prices.

The mean time between reference and admission was $71.3 \pm 46.3$ minutes therefore 1 hour 11 minutes and the mean time between admission and decision was $87.3 \pm 214.6$ minutes or 1 hour 27 minutes (Table 5). On the other hand [7] had found that the mean time between admission and decision was 52 minutes and that between the reference and admission was 32 minutes. This would be justified by the poverty that prevails in our environment since it is the patient and/or his family who must cover the cost of transportation, the purchase of the sheet and the operating kit, but religious beliefs and the fear of intervention can also come into play.

The time between decision and intervention was on mean $55.3 \pm 8.9$ minutes (in $86.4 \%$ of cases it was less than 60 minutes) and the mean duration of the intervention was $63.5 \pm 21.2$ minutes (in $51.5 \%$ this duration was greater than or equal to 60 minutes) (Table 5). These results differ from those found by [10] according to which the mean time between the decision and the start of the cesarean section was $26.4 \pm 5.48$ minutes and the mean duration of the intervention was $57.52 \pm 12.14$ minutes. This would be due to the scarcity of specialized labor and the material organization which is lame in under-equipped environments like ours.

Cesarean section was performed by the general practitioner in $98.1 \%$ (Table 6). On the other hand [15] found in his series that $46.7 \%$ of cesareans were performed by obstetricians. This could affect the quality of the cesarean section in our community.

Most cesarean sections were performed under general anesthesia at $98.1 \%$ (Table 6). These results are slightly higher than de [15] who found that general anesthesia was more used at $94.9 \%$. [11] found that in 36.5\% spinal anesthesia was used in their series. This would be a coincidence as dependent on one surgeon to another.

98.1\% of these were completed without incident, and $97.1 \%$ of the blood was not available. The operating kit was available in $97.1 \%$ of cases (Table 6). These results are inconsistent with those found in [11] [15], according to which the operating kit was $85 \%$ available. This would be justified by the fact that our study was carried out in the HGR which offer the Complementary Package of activities and must prepare accordingly.

With regard to antibiotic prophylaxis, $73.8 \%$ of respondents did not benefit from it, these results are not a corollary of those found by [11] according to which all the operated patients had benefited from antibiotic prophylaxis made of $2 \mathrm{~g}$ of ampicillin. This would be caused by the untimely stock-outs that we know in the HGR of our environment

Postoperative follow-up was $8.7 \%$ complicated (Table 6). These results are 
lower than those found by [8] according to whom in the postoperative phase $18.8 \%$ of those surveyed had complications. This is all the same affirmed by (16) who had found in their series that the operating suite was complicated with the RR: $2.46[1.32-4.59] \mathrm{p}=0.003$. This would be justified by the fact that these studies were carried out in different environments where the conditions surrounding the cesarean section are different.

At $100 \%$ the maternal prognosis was good (Table 6). On the other hand [16] had found that in emergency the maternal and fetal prognosis is grim than in the caesareans scheduled at the Brazzaville university hospital. As for [12], the maternal death rate was $17.1 \%$ compared to uterine ruptures, $2.0 \%$ compared to cesarean sections. This would be justified by the fact that our study is more recent which made it possible to adapt the preoperative, per and postoperative care of women undergoing cesarean section to current technology to minimize the risk of death.

Caesarized women were hospitalized on mean for $18.3 \pm 6.4$ days $(100 \%$ of our respondents had been hospitalized for 7 days or more) (Table 6).

Regarding the condition of the newborns at birth, $97.1 \%$ of them were in good condition, among them $63.1 \%$ were not resuscitated (Table 7). In contrast [8] found in their series that the perinatal mortality rate was high (15.9\%). This proves the significant advances observed in the fight against maternal and infant mortality in our environment.

The mean weight of the newborns was $3244.8 \pm 729.3$ grams (those with normal weight led with 78.6\%) (Table 7). On the other hand [17] had found that the mean weight in their series was $2286 \pm 267$ grams (the children of low weight represented $60 \%$ ). This is justified by the fact that his sample consisted of twin pregnancies for which women were cesarized.

Most of these newborns did not have a congenital malformation in $99.0 \%$ of cases and the prognosis of the newborn was satisfactory at $79.6 \%$ Table 7). On the other hand [16] had found no case of malformation and that in emergency the maternal and fetal prognosis was grim only in the caesareans scheduled at the CHU of Brazzaville.

$51.5 \%$ of the caesareans had a fairly good quality while $45.6 \%$ had an average quality (Table 8).

\subsection{Bi-Varied Analyses}

In our series, if the operating room was available, the probability of having an average quality cesarean was 90 times higher $(p<0.001), 14$ times higher in the case of a mother and child alive than in the death of the child $(\mathrm{p}<0.001)$ and 4.3 times weak or fairly good with an ineffective kit $(\mathrm{p}<0.001)$ (Table 12). On the other hand [8] found that the cesarean was not of good quality, the post-operative follow-up was not satisfactory, the delay in the decision of the cesarean and in the intervention. As for [7], found for his series that the indications were abusive, the post-operative follow-up insufficient and the maternal and perinatal mortal- 
ity high.

\section{Conclusions}

After collecting and analyzing data, we arrived at the results according to which:

The Caesarean section rate is $8.9 \%$;

The fairly good, weak and average quality cesarean section represented $51.5 \%$, $2.9 \%$ and $45.6 \%$ respectively.

The determinants of quality cesarean were: the availability of the operating room, mother-child prognosis and the availability of the operating kit. These results will help decision-makers in health matters to train and retrain health personnel, in particular midwives, on the benefits of prenatal consultation and its objectives, timely referral, and on the quality of work supervision.

\section{Conflicts of Interest}

The authors declare no conflicts of interest regarding the publication of this paper.

\section{References}

[1] United Nations Document (2015) Eradicate Poverty: MDGs.

[2] World Health Organization (2006) For a Safer Pregnancy. A Health Strategy Aimed at Reducing Maternal and Perinatal Mortality and Morbidity. Geneva, WHO/RHR/00.6.

[3] Berland, M. (1986) The Risks of the Cesarean Operation. French Review Gyneco-Obstetrics, 4, 5-12.

[4] Deneux-Tharaux, C. (2012) Cicatricial Uterus: Epidemiological Aspects. The Journal de Gynécologie Obstétrique et Biologie de la Reproduction, 50, 400.

[5] Blondel, B., Le long, N., Kermarrec, M. and Goffinet, F. (2012) Perinatal Health in Mainland France from 1995 to 2010. Results of National Perinatal Surveys. The Journal de Gynécologie Obstétrique et Biologie de la Reproduction, 9, 16-17.

[6] Say, L., Chou, D., Gemmill, A., Tunçalp, O., Moller, A.B. and Daniels, J. (2014) Global Causes of Maternal Death a WHO Systematic Analysis. Lancet Global Health, 2, e323-e333. https://doi.org/10.1016/S2214-109X(14)70227-X

[7] Chebou, K. (2008) The Determinants of Quality Caesarean Section at the Somine Dolo Hospital in Mopti from December 01, 2005 to November 30, 2006 on 299 Cases in the Republic of Mali.

[8] Ouedrago, C., Zougrana, T., Dao, B., Dujardin, B. and Kone, B. (2001) The Quality Cesarean Section at the Yalgado Ouedrago Hospital Center in Ouagadougou. Analysis of the Determinants Concerning 478 Cases Brought Up in the Gynecology and Obstetrics Department. Black African Medicine 2001. Collected in the Obstetrics Gynecology Department. Black African Medicine.

[9] Akilimali, P.Z., Nzau, N.E., Urbano, P. and Kaba, D.K. (2015) Predictors of Cesarean Delivery at the General Referral Hospital of Kinkanda at Matadi. Le Mali Medical, 30, 25-32.

[10] Biayi Mikenji, J., Mumba Mukandila, A., Kitengie Kapila, R., Kabila Ilunga, C., Kamonayi Mulumba, J., Mulumba, J. and Lumbala Tshimpanga, T. (2017) Emergency Cesarean in Semi-Urban Areas: Prevalence, Some Determinants and Indications by Applying Luscas Colors to HGR Kabinda. Ground Floor. Rev. Med Gds lakes, 9 
40-46.

[11] Some Der, A., Ouattara, S., Barro, D., Traoré, A., Bamabara, M. and Dao, B. (2005) Audit of Caesareans in African Environment. Rwandan Medical Review, 68, 34, 36.

[12] Sepou, A., Yanza, M.C., Nguembi, E., Ngbale, R., Kouriah, G., Kouabosso, A. and Nalim, N. (1998) Study of 299 Cases of Caesareans Performed at the Bangui Community Hospital (Central African Republic). Black African Medicine, 2000, 47.

[13] Togora, M. (2004) The Quality Cesarean at CS Ref. C V of the District of Bamako from 2000-2002: About 2883 Cases, Mali.

[14] World Health Organization (1996) New Mortality Estimates. Weekly Epidemiological Record.

[15] Keita, N., Diallo, M.-S., Ijazy, Y., Barry, M.D. and Touré, B. (2014) Ruptures Uterines. About 155 Cases Observed in Conakry (Republic of Guinea). The Journal de Gynécologie Obstétrique et Biologie de la Reproduction, 18, 1041-1047.

[16] Mbongo, J.A., Butoyi, J.M., Papandi-Ikourou, A. and Iloki, L.H. (2016) Emergency Caesarian Sections in the University Teaching Hospital of Brazzaville. Health Sci. Say, 17, 2-3.

[17] Fourn, L., Fayomi, E.B. and Zohoun, T.H. (1999) Epidemiological Aspects of the Practice of Cesareans in Twin Births in Cotonou. Black African Medicine, 46, 4-9. 